\title{
Uniqueness of the Fredholm-Stiltjes Linear Integral Equations Solutions of the Third Kind
}

\author{
Aizat Toigonbaeva1, Avyt Asanov², Aisalkyn Kambarova1, Gumushai Obodoeva1, \\ Ularbek Moldoyarov ${ }^{3}$, Aibek Toktorbaev ${ }^{4}$, Aichurok Abdukadyr Kyzy'1, Zhypargul Abdullaeva ${ }^{5^{*}}$ (C) \\ ${ }^{1}$ Department of Mathematical Analysis, Osh State University, Osh, Kyrgyzstan \\ ${ }^{2}$ Mathematics Department, Kyrgyz Turkish Manas University, Bishkek, Kyrgyzstan \\ ${ }^{3}$ Department of Automated Systems and Digital Technologies, Osh State University, Osh, Kyrgyzstan \\ ${ }^{4}$ Department of Information Systems and Programming, Osh State University, Osh, Kyrgyzstan \\ ${ }^{5}$ Science and Research Department, Osh State University, Osh, Kyrgyzstan \\ Email: *jypar.science@oshsu.kg
}

How to cite this paper: Toigonbaeva, A., Asanov, A., Kambarova, A., Obodoeva, G., Moldoyarov, U., Toktorbaev, A., Kyzy, A.A. and Abdullaeva, Z. (2021) Uniqueness of the Fredholm-Stiltjes Linear Integral Equations Solutions of the Third Kind. Advances in Linear Algebra \& Matrix Theory, 11, 109-116.

https://doi.org/10.4236/alamt.2021.114008

Received: October 4, 2021

Accepted: November 12, 2021

Published: November 15, 2021

Copyright (c) 2021 by author(s) and Scientific Research Publishing Inc. This work is licensed under the Creative Commons Attribution International License (CC BY 4.0).

http://creativecommons.org/licenses/by/4.0/

\begin{abstract}
Integral equations theoretical parts and applications have been studied and investigated in previous works. In this work, results on investigations of the uniqueness of the Fredholm-Stiltjes linear integral equations solutions of the third kind were considered. Volterra integral equations of the first and third kind with smooth kernels were studied, and proof of the existence of a multiparameter family of solutions is described. Additionally, linear Fredholm integral equations of the first kind were investigated, for which Lavrent'ev regularizing operators were constructed.
\end{abstract}

\section{Keywords}

Solution, Integral Equations, Uniqueness, Fredholm-Stiltjes Linear Integral Equations, Third Kind

\section{Introduction}

The theoretical part and applications of integral equations have been studied and investigated in many different works. In particular, in [1], a survey of the results of investigations of Volterra integral equations of the second kind was considered. In [2], Volterra integral equations of the first and third kind with smooth kernels are studied, where a proof of the existence of a multiparameter family of solutions is given. In [3], linear Fredholm integral equations of the first kind were investigated, for which Lavrent'ev regularizing operators were constructed. In [4], a theory is presented and numerical methods are used for solving non- 
classical Volterra integral equations of the first kind with differentiable and nonzero kernels on the diagonal. In papers [4] [5] [6] [7] applications of non-classical Volterra integral equations of the first kind in various applied problems are given. In [8], the Lavrent'ev regularization method is used for Volterra integral equations of the first kind with smooth and nonzero kernels on the diagonal and differentiable solutions, for which an approximate solution is constructed. In works [9] [10], sufficient conditions for the uniqueness of solutions were obtained and the problems of regularization of solutions to systems of linear and nonlinear Volterra integral equations of the first and third kind were investigated. In [11], a unique theorem for solutions is proved and a regularizing operator is found for solving a system of Volterra linear integral equations of the third kind. In works [12] [13], a new approach was used to study the existence and uniqueness of solutions of scalar Fredholm integral equations of the third kind with multipoint singularities and their systems. In [14], results are given on the Volterra integral equations of the first kind. In [15], derivative concept with respect to an increasing function was introduced, using linear Fredholm-Stiltjes integral equations of the first kind in [16] [17] [18]. Numerical solution of the Fredholm and Volterra Integral equations by using modified Bernstein-Kantorovich operators [19], second kind [20], and third kind of nonlinear Volterra-Stieltjes integral equations with the solution by Lavrentyev regularizing operator were also described [21].

In this paper, based on concept of derivatives with respect to an increasing function, using the method of nonnegative quadratic forms, we establish sufficient conditions for the uniqueness of a solution for Fredholm-Stiltjes integral equations of the third kind (1).

We will consider the equation

$$
m(t) u(t)+\int_{a}^{b} K(t, s) u(s) \mathrm{d} \phi(s)=f(t), t \in[a, b],
$$

here $m(t), \phi(t), K(t, s)$ and $f(t)$-are known functions, $\phi(t)$-increasing continuous function on $[a, b], m(t) \in C[a, b], 0 \leq m(t)$ at all conditions $t \in[a, b]$ and $m(t)$ is equal to zero at least at one point of the segment $[a, b]$,

$$
K(t, s)= \begin{cases}A(t, s), & a \leq s \leq t \leq b, \\ B(t, s), & a \leq t \leq s \leq b .\end{cases}
$$

It is assumed that $A(t, s)$ and $B(t, s)$ is continuous function on $\{(t, s): a \leq s \leq t \leq b\}$, and $\{(t, s): a \leq t \leq s \leq b\}$, solution $u(t)$ searched in $C[a, b]$.

Let's assume:

1) $H(t, s)=A(t, s)+B(s, t)$ and $H_{\phi(t) \phi(s)}^{\prime \prime}(t, s)$ are continuous functions in the domain $G=\{(t, s): a \leq s \leq t \leq b\}, H_{\phi(t)}^{\prime}(t, a)$ and $H_{\phi(t)}^{\prime}(b, t)$, and are continuous functions in $[a, b]$, here;

$$
H_{\phi(t)}^{\prime}(t, s)=\lim _{\Delta \rightarrow 0} \frac{H(t+\Delta, s)-H(t, s)}{\phi(t+\Delta)-\phi(t)} ;
$$


2) $H(b, a) \geq 0, H_{\phi(t)}^{\prime}(t, a) \leq 0$ and $H_{\phi(t)}^{\prime}(b, t) \geq 0, \forall t \in[a, b]$, $H_{\phi(t) \phi(s)}^{\prime \prime}(t, s) \leq 0, \quad \forall(t, s) \in G$;

3) $H_{\phi(t)}^{\prime}(t, a)<0$ for $t \in[a, b]$; or $H_{\phi(t)}^{\prime}(b, t)>0$ for $t \in[a, b]$; or $H_{\phi(t) \phi(s)}^{\prime \prime}(t, s)<0$ for $(t, s) \in G$;

4) $m(t)>0$ for $t \in[a, b]$.

If taking into account (2), then Equation (1) have the following form

$$
m(t) u(t)+\int_{a}^{t} A(t, s) u(s) \mathrm{d} \phi(s)+\int_{t}^{b} B(t, s) u(s) \mathrm{d} \phi(s)=f(t) .
$$

By multiplying $u(t)$ to Equation (3) and integrating according to Stieltjes over the $a \leq t \leq b$ area, we will have

$$
\begin{aligned}
& \int_{a}^{b} m(t) u^{2}(t) \mathrm{d} t+\int_{a}^{b} \int_{a}^{t} A(t, s) u(s) u(t) \mathrm{d} \phi(s) \mathrm{d} \phi(t) \\
& +\int_{a}^{b} \int_{t}^{b} B(t, s) u(s) u(t) \mathrm{d} \phi(t) \mathrm{d} \phi(s)=\int_{a}^{b} f(t) u(t) \mathrm{d} \phi(t) .
\end{aligned}
$$

We use the generalized Dirichlet formula:

$$
\begin{gathered}
\int_{a}^{b} m(t) u^{2}(t) \mathrm{d} t+\int_{a}^{b} \int_{a}^{t} A(t, s) u(s) u(t) \mathrm{d} \phi(s) \mathrm{d} \phi(t) \\
+\int_{a}^{b} \int_{a}^{s} B(t, s) u(s) u(t) \mathrm{d} \phi(s) \mathrm{d} \phi(t)=\int_{a}^{b} f(t) u(t) \mathrm{d} \phi(t), \\
\text { i.e. } \int_{a}^{b} m(t) u^{2}(t) \mathrm{d} t+\int_{a}^{b} \int_{a}^{t}[A(t, s)+B(s, t)] u(s) u(t) \mathrm{d} \phi(s) \mathrm{d} \phi(t)=\int_{a}^{b} f(t) u(t) \mathrm{d} \phi(t) .
\end{gathered}
$$

The designation is made for $H(t, s)=A(t, s)+B(s, t)$, then

$$
\int_{a}^{b} m(t) u^{2}(t) \mathrm{d} t+\int_{a}^{b} \int_{a}^{t} H(t, s) u(s) \mathrm{d} \phi(s) u(t) \mathrm{d} \phi(t)=\int_{a}^{b} f(t) u(t) \mathrm{d} \phi(t) .
$$

After introduction of notation

$$
Z(t, s)=\int_{s}^{t} u(v) \mathrm{d} \phi(v),
$$

It turns out that $\mathrm{d}_{\phi(s)} Z(t, s)=-u(s) \mathrm{d} \phi(s)$, also, $u(t) \mathrm{d} \phi(t)=\mathrm{d}_{\phi(t)} Z(t, s)$.

By multiplying $2 Z(t, s)$ we obtain

$$
\begin{gathered}
2 Z(t, s) u(t) \mathrm{d} \phi(t)=2 Z(t, s) \mathrm{d}_{\phi(t)} Z(t, s)=\mathrm{d}_{\phi(t)} Z^{2}(t, s), \\
Z(t, s) u(t) \mathrm{d} \phi(t)=\frac{1}{2} \mathrm{~d}_{\phi(t)} Z^{2}(t, s),
\end{gathered}
$$

In this work, based on the concept of the derivative with respect to an increasing function, using the method of nonnegative quadratic forms, for the first time, sufficient conditions for uniqueness of a solution for Fredholm-Stiltjes integral equations of the third kind were established (1).

By applying (7), (8), and the method of integration by parts and the generalized Dirichlet formula, from (6) we have 


$$
\begin{aligned}
& \int_{a}^{b} \int_{a}^{t} H(t, s) u(s) \mathrm{d} \phi(s) u(t) \mathrm{d} \phi(t) \\
& =-\int_{a}^{b}\left[\int_{a}^{t} H(t, s) \mathrm{d}_{\phi(s)} Z(t, s)\right] u(t) \mathrm{d} \phi(t) \\
& =-\int_{a}^{b}\left[\left.H(t, s) Z(t, s)\right|_{a} ^{t}-\int_{a}^{t} H_{\phi(s)}^{\prime} Z(t, s) \mathrm{d} \phi(s)\right] u(t) \mathrm{d} \phi(t) \\
& =\int_{a}^{b} H(t, a) Z(t, a) u(t) \mathrm{d} \phi(t)+\int_{a}^{b} \int_{a}^{t} H_{\phi(s)}^{\prime} Z(t, s) \mathrm{d} \phi(s) u(t) \mathrm{d} \phi(t) \\
& =\frac{1}{2} \int_{a}^{b} H(t, a) \mathrm{d}_{\phi(t)} Z^{2}(t, a)+\int_{a}^{b} \int_{s}^{b} H_{\phi(s)}^{\prime}(t, s) Z(t, s) u(t) \mathrm{d} \phi(t) \mathrm{d} \phi(s) \\
& =\left.\frac{1}{2} H(t, a) Z^{2}(t, a)\right|_{a} ^{b}-\frac{1}{2} \int_{a}^{b} H_{\phi(t)}^{\prime}(t, a) Z^{2}(t, a) \mathrm{d} \phi(t) \\
& +\frac{1}{2} \int_{a}^{b}\left[\int_{s}^{b} H_{\phi(s)}^{\prime}(t, s) \mathrm{d}_{\phi(t)} Z^{2}(t, s)\right] \mathrm{d} \phi(s) \\
& =\frac{1}{2} H(b, a) Z^{2}(b, a)-\frac{1}{2} \int_{a}^{b} H_{\phi(t)}^{\prime}(t, a) Z^{2}(t, a) \mathrm{d} \phi(t) \\
& +\frac{1}{2} \int_{a}^{b} H_{\phi(s)}^{\prime}(b, s) Z^{2}(b, s) \mathrm{d} \phi(s)-\frac{1}{2} \int_{a}^{b} \int_{a}^{t} H_{\phi(s) \phi(t)}^{\prime \prime}(t, s) Z^{2}(t, s) \mathrm{d} \phi(s) \mathrm{d} \phi(t) \\
& =\frac{1}{2} H(b, a)\left[\int_{a}^{b} u(s) \mathrm{d} \phi(s)\right]^{2}-\frac{1}{2} \int_{a}^{b} H_{\phi(t)}^{\prime}(t, a)\left[\int_{a}^{t} u(s) \mathrm{d} \phi(s)\right]^{2} \mathrm{~d} \phi(t) \\
& +\frac{1}{2} \int_{a}^{b} H_{\phi(s)}^{\prime}(b, s)\left[\int_{s}^{b} u(\xi) \mathrm{d} \phi(\xi)\right]^{2} \mathrm{~d} \phi(s) \\
& -\frac{1}{2} \int_{a}^{b} \int_{a}^{t} H_{\phi(t) \phi(s)}^{\prime \prime}\left[\int_{s}^{t} u(\xi) \mathrm{d} \phi(\xi)\right]^{2} \mathrm{~d} \phi(s) \mathrm{d} \phi(t),
\end{aligned}
$$

here $Z(t, t)=0$.

From Equation (6) we obtaining

$$
\begin{aligned}
& \int_{a}^{b} \int_{a}^{t} H(t, s) u(s) \mathrm{d} \phi(s) u(t) \mathrm{d} \phi(t) \\
& =-\int_{a}^{b}\left[\int_{a}^{t} H(t, s) \mathrm{d}_{\phi(s)} Z(t, s)\right] u(t) \mathrm{d} \phi(t) \\
& =-\int_{a}^{b}\left[\left.H(t, s) Z(t, s)\right|_{a} ^{t}-\int_{a}^{t} H_{\phi(s)}^{\prime} Z(t, s) \mathrm{d} \phi(s)\right] u(t) \mathrm{d} \phi(t) \\
& =\int_{a}^{b} H(t, a) Z(t, a) u(t) \mathrm{d} \phi(t)+\int_{a}^{b} \int_{a}^{t} H_{\phi(s)}^{\prime} Z(t, s) \mathrm{d} \phi(s) u(t) \mathrm{d} \phi(t) \\
& =\frac{1}{2} \int_{a}^{b} H(t, a) \mathrm{d}_{\phi(t)} Z^{2}(t, a)+\int_{a}^{b} \int_{s}^{b} H_{\phi(s)}^{\prime}(t, s) Z(t, s) u(t) \mathrm{d} \phi(t) \mathrm{d} \phi(s) \\
& =\left.\frac{1}{2} H(t, a) Z^{2}(t, a)\right|_{a} ^{b}-\frac{1}{2} \int_{a}^{b} H_{\phi(t)}^{\prime}(t, a) Z^{2}(t, a) \mathrm{d} \phi(t) \\
& +\frac{1}{2} \int_{a}^{b}\left[\int_{s}^{b} H_{\phi(s)}^{\prime}(t, s) \mathrm{d}_{\phi(t)} Z^{2}(t, s)\right] \mathrm{d} \phi(s)
\end{aligned}
$$




$$
\begin{aligned}
= & \frac{1}{2} H(b, a) Z^{2}(b, a)-\frac{1}{2} \int_{a}^{b} H_{\phi(t)}^{\prime}(t, a) Z^{2}(t, a) \mathrm{d} \phi(t) \\
& +\frac{1}{2} \int_{a}^{b} H_{\phi(s)}^{\prime}(b, s) Z^{2}(b, s) \mathrm{d} \phi(s)-\frac{1}{2} \int_{a}^{b} \int_{a}^{t} H_{\phi(s) \phi(t)}^{\prime \prime}(t, s) Z^{2}(t, s) \mathrm{d} \phi(s) \mathrm{d} \phi(t) \\
= & \frac{1}{2} H(b, a)\left[\int_{a}^{b} u(s) \mathrm{d} \phi(s)\right]^{2}-\frac{1}{2} \int_{a}^{b} H_{\phi(t)}^{\prime}(t, a)\left[\int_{a}^{t} u(s) \mathrm{d} \phi(s)\right]^{2} \mathrm{~d} \phi(t) \\
& +\frac{1}{2} \int_{a}^{b} H_{\phi(s)}^{\prime}(b, s)\left[\int_{s}^{b} u(\xi) \mathrm{d} \phi(\xi)\right]^{2} \mathrm{~d} \phi(s) \\
& -\frac{1}{2} \int_{a}^{b} \int_{a}^{t} H_{\phi(t) \phi(s)}^{\prime \prime}\left[\int_{s}^{t} u(\xi) \mathrm{d} \phi(\xi)\right]^{2} \mathrm{~d} \phi(s) \mathrm{d} \phi(t),
\end{aligned}
$$

If we take into account (9), then from (6) we will have

$$
\begin{aligned}
& \int_{a}^{b} m(t) u^{2}(t) \mathrm{d} t+\frac{1}{2} H(b, a)\left[\int_{a}^{b} u(s) \mathrm{d} \phi(s)\right]^{2} \\
& -\frac{1}{2} \int_{a}^{b} H_{\phi(t)}^{\prime}(t, a)\left[\int_{a}^{t} u(s) \mathrm{d} \phi(s)\right]^{2} \mathrm{~d} \phi(t) \\
& +\frac{1}{2} \int_{a}^{b} H_{\phi(s)}^{\prime}(b, s)\left[\int_{s}^{b} u(\xi) \mathrm{d} \phi(\xi)\right]^{2} \mathrm{~d} \phi(s) \\
& -\frac{1}{2} \int_{a}^{b} \int_{a}^{t} H_{\phi(t) \phi(s)}^{\prime \prime}\left[\int_{s}^{t} u(\xi) \mathrm{d} \phi(\xi)\right]^{2} \mathrm{~d} \phi(s) \mathrm{d} \phi(t)=\int_{a}^{b} f(t) u(t) \mathrm{d} \phi(t) .
\end{aligned}
$$

Thus, (10) holds for all solutions of $u(t)$ Equation (1) under condition 1), if $f(t) \equiv 0$, then under conditions 2$), 3$ ) from (10) it follows:

$$
\begin{gathered}
\int_{a}^{t} u(s) \mathrm{d} \phi(s) \equiv 0 \quad \text { or } \int_{t}^{b} u(s) \mathrm{d} \phi(s) \equiv 0 \text { or } \int_{s}^{t} u(\xi) \mathrm{d} \phi(\xi) \equiv 0, \\
t, s \in[a, b], s<t .
\end{gathered}
$$

from here $u(t) \equiv 0$. The following theorem is proved.

\section{Theorem 1.}

If conditions 1)-4) are satisfied, then Equation (1) in $C[a, b]$ has at most one solution.

Example.

We consider the equation

$$
\begin{gathered}
t u(t)+\int_{0}^{1} K(t, s) u(s) \mathrm{d} \phi(s)=f(t), t \in[0,1], \text { where } \phi(t)=\sqrt{t}, t \in[0,1], \\
K(t, s)= \begin{cases}\frac{1}{2}(1-\sqrt{t})^{k}(\sqrt{s})^{m}, & 0 \leq t \leq s \leq 1, \\
\frac{1}{2}(1-\sqrt{s})^{k}(\sqrt{t})^{m}, & 0 \leq s \leq t \leq 1,\end{cases}
\end{gathered}
$$

In this case $m(t)=t, k>1, m>1$. Then 


$$
\begin{aligned}
H(t, s) & =A(t, s)+B(s, t) \\
& =\frac{1}{2}(1-\sqrt{t})^{k}(\sqrt{s})^{m}+\frac{1}{2}(1-\sqrt{t})^{k}(\sqrt{s})^{m} \\
& =(1-\sqrt{t})^{k}(\sqrt{s})^{m} .
\end{aligned}
$$

using the above formula (9) will lead to Equation (14). Applying (9) and taking into account (13), we have

$$
\begin{aligned}
& \int_{0}^{1} \int_{0}^{t}(1-\sqrt{t})^{k}(\sqrt{s})^{m} u(s) \mathrm{d} \phi(s) u(t) \mathrm{d} \phi(t) \\
& =-\frac{1}{2} \int_{0}^{1} \int_{0}^{t}\left(-m k(1-\sqrt{t})^{k-1}(\sqrt{s})^{m-1}\right)\left[\int_{s}^{t} u(\xi) \mathrm{d} \phi(\xi)\right]^{2} \mathrm{~d} \phi(s) \mathrm{d} \phi(t),
\end{aligned}
$$

In this case $H(1,0)=0, H_{\phi(t)}^{\prime}(t, 0)=0, H_{\phi(s)}^{\prime}(1, s)=0$,

$$
\begin{gathered}
H_{\phi(s) \phi(t)}^{\prime \prime}(t, s)=-m k(1-\sqrt{t})^{k-1}(\sqrt{s})^{m-1} \leq 0, \\
\forall(t, s) \in G=\{(t, s) \mid 0 \leq s \leq t \leq 1\} ; \\
H_{\phi(s) \phi(t)}^{\prime \prime}(t, s)=-m k(1-\sqrt{t})^{k-1}(\sqrt{s})^{m-1}<0,
\end{gathered}
$$

with almost all $(t, s) \in G=\{(t, s) \mid 0 \leq s \leq t \leq 1\}$.

This shows that all conditions of Theorem 1 are satisfied.

If $f(t)=0$ at all conditions of $t \in[0,1]$, then from (14) we will have

$$
\frac{1}{2} \int_{0}^{1} \int_{0}^{t}\left(-k m(1-\sqrt{t})^{k-1}(\sqrt{s})^{m-1}\right)\left[\int_{s}^{t} u(s) \mathrm{d} \phi(s)\right]^{2} \mathrm{~d} \phi(s) \mathrm{d} \phi(t)=0 .
$$

Equation (15) implies

$$
\int_{s}^{t} u(s) \mathrm{d} \phi(s)=0,(t, s) \in G
$$

It follows that $u(t)=0, t \in[0,1]$.

\section{Conclusion}

In summary, results of the Fredholm-Stiltjes linear integral equations solutions uniqueness of the third kind were described. The Volterra integral equations of the first and third kind with smooth kernels were studied, and proof of the existence of solutions is shown. The work was devoted to the study of uniqueness questions for linear Fredholm-Stiltjes integral equations of third kind. Sufficient conditions for uniqueness of solutions for the integral equations under study were established. In the future, it is planned to study the problems of constructing regularizing operators for Fredholm-Stiltjes integral third kind equations.

\section{Conflicts of Interest}

The authors declare no conflicts of interest regarding the publication of this paper. 


\section{References}

[1] Tselyuk, Z.B. (1977) Volterra Integral Equations. Results of Science and Technology. Mathematical Analysis, 15, 131-198.

[2] Magnitsky, N.A. (1979) Volterra Linear Integral Equations of the First and Third Kind. Journal of Computational Mathematics and Mathematical Physics, 19, 970-989. https://doi.org/10.1016/0041-5553(79)90166-6

[3] Lavrent'ev, M.M. (1959) On Integral Equations of the First Kind. Doklady Akademii Nauk SSSR, 127, 31-33.

[4] Apartsin, A.S. (1999) Non-Classical Volterra Equations of the First Kind. In: Theory and Numerical Methods, Nauka: Siberian Branch, Novosibirsk, p. 193.

[5] Apartsin, A.S., Karaulova, I.V., Markova, E.V. and Trufanov, V.V. (2005) Application of Volterra Integral Equations for Modeling Strategies for Technical Retrofitting of the Electric Power Industry. Electricity, 10, 69-75.

[6] Apartsin, A.S. and Sidler, I.V. (2018) Study of Volterra Test Equations of the First Kind in Integral Models of Developing Systems. Proceedings of the Institute of Mathematics and Mechanics, Ural Branch of the Russian Academy of Sciences, 24, 24-33.

[7] Glushkov, V.M., Ivanov, V.V. and Yanenko, V.M. (1983) Modeling of Developing Systems. Nauka, Moscow.

[8] Denisov, A.M. (1975) On the Approximate Solution of the Volterra Equation of the First Kind. Journal of Computational Mathematics and Mathematical Physics, 15, 1053-1056. https://doi.org/10.1016/0041-5553(75)90185-8

[9] Imanaliev, M.I. and Asanov, A. (1989) On Solutions of Systems of Nonlinear Volterra Integral Equations of the First Kind. Doklady Akademii Nauk SSSR, 309, 1052-1055.

[10] Imanaliev, M.I. and Asanov, A. (2007) Regularization and Uniqueness of Solutions of Systems of Nonlinear Volterra Integral Equations of the Third Kind. Doklady $R A N, 415,14-17$.

[11] Imanaliev, M.I. and Asanov, A. (2010) On Solutions of Systems of Fredholm Linear Integral Equations of the Third Kind. Doklady RAN, 430, 1-4.

[12] Imanaliev, M.I., Asanov, A. and Asanov, R.A. (2018) On a Class of Systems of Linear and Nonlinear Fredholm Integral Equations of the Third Kind with Multipoint Singularities. Differential Equations, 54, 387-397. https://doi.org/10.1134/S0012266118030096

[13] Asanov, A., Matanova, K. and Asanov, R. (2017) A Class of Linear and Nonlinear Fredholm Integral Equations of the Third Kind. Kuwait Journal of Science, 44, $17-28$.

[14] Lamm, R.K. (2000) A Survey of Regularization Methods for First Kind Volterra Equations, Surveys on Solution Methods for Inverse Problems. Springer, Vienna, 53-82. https://doi.org/10.1007/978-3-7091-6296-5_4

[15] Asanov, A. (2001) Derivative of a Function with Respect to an Increasing Function. Journal of Natural Sciences, 1, 18-64.

[16] Toygonbaeva, A.K., Asanov, A. and Kalimbetov, B. (2012) On One Class of Fredholm-Stiltjes Linear Integral Equations of the First Kind. Bulletin of Karaganda University, Mathematics Series, 4, 3-6.

[17] Toygonbaeva, A.K. and Asanov, A. (2012) On a Class of Systems of Fredholm-Stiltjes Integral Equations of the First Kind with a Discontinuous Kernel. Stu- 
dies on Integro-Differential Equations. Bishkek. Ilim, 45, 50-55.

[18] Toigonbaeva, A.K. and Asanov, A. (2019) The Choice of Regularization Parameter of Solutions of Linear Fredholm-Stieltjes Integral Equations of the First Kind. Science, New Technologies and Innovations of Kyrgyzstan, Bishkek, 6, 3-8.

[19] Buranay, S.C., Özarslan, M.A. and Falahhesar, S.S. (2021) Numerical Solution of the Fredholm and Volterra Integral Equations by Using Modified Bernstein-Kantorovich Operators. Mathematics, 9, 1193. https://doi.org/10.3390/math9111193

[20] Mosa, G.A., Abdou, M.A. and Rahby, A.S. (2021) Numerical Solutions for Nonlinear Volterra-Fredholm Integral Equations of the Second Kind with a Phase Lag. AIMS Mathematics, 6, 8525-8543. https://doi.org/10.3934/math.2021495

[21] Bedelova, N., Asanov, A., Orozmamatova, Z. and Abdullaeva, Z. (2021) Regularization and Choice of the Parameter for the Third Kind Nonlinear Volterra-Stieltjes Integral Equation Solutions. International Journal of Modern Nonlinear Theory and Application, 10, 81-90. https://doi.org/10.4236/ijmnta.2021.102006 